\title{
HAUSDORFF $m$ REGULAR AND RECTIFIABLE SETS IN $n$-SPACE $\left({ }^{1}\right)$
}

BY

PERTTI MATTILA

\begin{abstract}
The purpose of this paper is to prove the following theorem: If $E$ is a subset of Euclidean $n$-space and if the $m$-dimensional Hausdorff density of $E$ exists and equals one $H^{m}$ almost everywhere in $E$, then $E$ is countably $\left(H^{m}, m\right)$ rectifiable. Here $H^{m}$ is the $m$-dimensional Hausdorff measure. The proof is a generalization of the proof given by J. M. Marstrand in the special case $n=3, m=2$.
\end{abstract}

1. Notation and terminology. Throughout the whole paper $m$ and $n$ will be fixed integers such that $1 \leqslant m \leqslant n-1$. The $n$-dimensional Euclidean space will be denoted by $R^{n}$. For $x, y \in R^{n}, x \cdot y$ is the usual inner product of $x$ and $y$, and $|x|$ is the norm of $x$. If $E \subset R^{n}$ and $x \in R^{n}$, then $d(E)$ is the diameter of $E, d(x, E)$ is the distance from $x$ to $E$ and, if $\delta>0$, $E(\delta)=\left\{x \in R^{n}: d(x, E) \leqslant \delta\right\}$. The boundary of $E$ is denoted by $\partial E$. For $a \in R^{n}$ and $0<r<\infty$, we let $U(a, r)=\left\{x \in R^{n}:|a-x|<r\right\}$ and $B(a, r)=$ $\left\{x \in R^{n}:|a-x| \leqslant r\right\}$. We denote by $\alpha(m)$ the volume of the $m$-dimensional unit ball.

If $k$ is an integer such that $0 \leqslant k \leqslant n$, then $G(n, k)$ stands for the set of all $k$-dimensional linear subspaces of $R^{n}$. If $a \in R^{n}$, then $A(a, n, k)$ is the set of all $k$-dimensional affine subspaces through $a$. For $V \in A(a, n, k)$, the orthogonal projection from $R^{n}$ onto $V$ will be denoted $P_{V}$.

The $m$-dimensional Hausdorff measure in $R^{n}$ will be denoted by $H^{m}$ The $m$-dimensional Hausdorff lower density, upper density and density of $E \subset$ $R^{n}$ at $x \in R^{n}$ are defined by

$$
\begin{aligned}
& \Theta_{*}^{m}(E, x)=\liminf _{r \rightarrow 0+} \alpha(m)^{-1} r^{-m} H^{m}[E \cap B(x, r)], \\
& \Theta^{* m}(E, x)=\limsup _{r \rightarrow 0+} \alpha(m)^{-1} r^{-m} H^{m}[E \cap B(x, r)],
\end{aligned}
$$

Received by the editors February 4, 1974.

AMS (MOS) subject classifications (1970). Primary 28A05, 28 A75.

Key words and phrases. Hausdorff measure, density, regular set, rectifiable set.

(1) Most of this work was done while the author was visiting Indiana University in 1972-73 sponsored by United States Government PL 87-256 Graduate Study Grant. This research was also supported by a grant from the Finnish Cultural Foundation. 


$$
\Theta^{m}(E, x)=\lim _{r \rightarrow 0+} \alpha(m)^{-1} r^{-m} H^{m}[E \cap B(x, r)] \text {. }
$$

If $\underset{1}{E}$ is $H^{m}$ measurable and $0<H^{m}(E)<\infty$, we say that $E$ is an $m$-set. If $E$ is an $m$-set and $\Theta^{m}(E, x)=1$ for $H^{m}$ a.e. $x \in E$, we say that $E$ is $m$ regular. $E$ is $m$ rectifiable if there exist bounded sets $A_{i} \subset R^{m}$ and Lipschitzian maps $f_{i}: R^{m} \rightarrow R^{n}$ such that $H^{m}\left[E \backslash \bigcup_{i=1}^{\infty} f_{i}\left(A_{i}\right)\right]=0$. We say that $E$ is purely $m$ unrectifiable if it contains no $m$ rectifiable set $F$ with $H^{m}(F)>0$. These concepts are the same as countably $\left(H^{m}, m\right)$ rectifiable and purely $\left(H^{m}\right.$, $m)$ unrectifiable in $[2,3.2 .14]$.

2. The main theorem. In this paper we prove the following theorem:

2.1. THEOREM. If $E \subset R^{n}$ and $\Theta^{m}(E, x)=1$ for $H^{m}$ a.e. $x \in E$, then $E$ is $m$ rectifiable.

This was proved by Marstrand [3] in the special case $n=3, m=2$, and the present proof is a generalization of Marstrand's proof. In the case $m=1$, a more general result has been proved by Morse and Randolph [5] for $n=2$ and by Moore [4] for all integers $n \geqslant 2$.

I want to thank Dr. W. P. Ziemer, who suggested this problem.

3. Density theorems. We state three results which will be used later on. The first follows from $[2,2.10 .17(3)$ and 2.10.18(3)], the second from [2, 2.10.19(5) and (4)] and the third is a corollary to the second.

3.1. THEOREM. If $E \subset R^{n}$ and $H^{m}(E)<\infty$, then

$$
\lim _{\delta \rightarrow 0+}\left[\sup \left\{\alpha(m)^{-1} 2^{m} d(S)^{-m} H^{m}(E \cap S): x \in S, d(S)<\delta\right\}\right]=1
$$

for $H^{m}$ a.e. $x \in E$.

3.2. THEOREM. If $E \subset R^{n}$ and $H^{m}(E)<\infty$, then $\Theta^{* m}(E, x) \leqslant 1$ for $H^{m}$ a.e. $x \in R^{n}$. If, in addition, $E$ is $H^{m}$ measurable, then $\Theta^{m}(E, x)=0$ for $H^{m}$ a.e. $x \in R^{n} \backslash E$.

3.3. THEOREM. If $E \subset F \subset R^{n}, F$ is $m$ regular and $E$ is an $m$-set, then $E$ is $m$ regular.

4. Directional properties of $m$ regular sets. Given an $m$ regular set $E$, we are in this section mainly concerned with finding an $m$-subset of $E$ with certain directional properties. The sets $B(a, r) \cap V(\lambda), a \in E, V \in A(a, n, m)$, $r>0$ and $0<\lambda<1$, will play an important role. They correspond to the sections $\operatorname{Se}(a ; i, \lambda, r)$ in [3].

4.1. LEMmA. Suppose that $E$ is an $m$ regular subset of $R^{n}, \eta>0$ and 
$\delta>0$. Then there are a positive number $d_{1}$ and an m-set $E_{1} \subset E$ such that $H^{m}\left(E \backslash E_{1}\right)<\eta$ and $2 a-b \in E(\delta|a-b|)$ whenever $a, b \in E_{1}$ and $|a-b|<d_{1}$.

Proof. We may assume that $\delta<1$. Let $0<\epsilon<1 / 3$. By 3.1 and the regularity of $E$, we can find a positive number $d_{1}$ and an $m$-set $E_{1} \subset E$ such that $H^{m}\left(E \backslash E_{1}\right)<\eta$ and

(1) $H^{m}(E \cap S) \leqslant\left(1+\epsilon^{m}\right) \alpha(m) 2^{-m} d(S)^{m}$ if $E_{1} \cap S \neq \varnothing$ and $d(S)<2 d_{1}$,

(2) $H^{m}[E \cap B(a, r)]>\left(1-\epsilon^{m}\right) \alpha(m) r^{m}$ if $a \in E_{1}$ and $0<r<d_{1}$.

Let $a, b \in E_{1}$ be such that $0<|a-b|=\rho<d_{1}$ and denote

$$
\begin{gathered}
b^{\prime}=2 a-b, \quad c=3 \epsilon a+(1-3 \epsilon) b, \quad c^{\prime}=3 \epsilon a+(1-3 \epsilon) b^{\prime}, \\
A=B[a,(1-3 \epsilon) \rho], \quad B=B(b, 2 \epsilon \rho), \quad C=B\left[c^{\prime}, \delta(1-3 \epsilon) \rho / 2\right] .
\end{gathered}
$$

Then $c, c^{\prime} \in \partial A$ and $\left|c-c^{\prime}\right|=2(1-3 \epsilon) \rho$. It is easy to see that if we put $M=\sup \{|x-c| /(1-3 \epsilon) \rho: x \in A \backslash C\}$, then $M<2$ and $M$ depends only on $\delta$ and $n$. We choose $\epsilon$ so small that

$$
\begin{gathered}
\delta(1-3 \epsilon) / 2+3 \epsilon<\delta, \\
M(1-3 \epsilon)+5 \epsilon<2(1-3 \epsilon) .
\end{gathered}
$$

Set $S=(A \cup B) \backslash C$. Then (3) implies that $A \cap C \subset B\left(b^{\prime}, \delta \rho\right)$ and, since $\epsilon<$ $1 / 3$, (4) implies that $d(S) \leqslant 2(1-3 \epsilon) \rho<2 d_{1}$. Moreover, by assumption $\delta<$ 1 , it follows that $B \cap C=\varnothing$ and $b \in E_{1} \cap S$. Hence we get from (1)

$$
H^{m}(E \cap S) \leqslant\left(1+\epsilon^{m}\right) \alpha(m)(1-3 \epsilon)^{m} \rho^{m}
$$

and (2) gives

$$
H^{m}(E \cap A)>\left(1-\epsilon^{m}\right) \alpha(m)(1-3 \epsilon)^{m} \rho^{m}
$$

and

$$
H^{m}(E \cap B)>\left(1-\epsilon^{m}\right) \alpha(m) 2^{m} \epsilon^{m} \rho^{m}
$$

Hence

$$
\begin{aligned}
H^{m}\left[E \cap B\left(b^{\prime}, \delta \rho\right)\right] & \geqslant H^{m}(E \cap A \cap C) \\
& =H^{m}(E \cap A)+H^{m}(E \cap B)-H^{m}(E \cap S) \\
& >2 \alpha(m) \epsilon^{m} \rho^{m}\left[2^{m-1}\left(1-\epsilon^{m}\right)-(1-3 \epsilon)^{m}\right],
\end{aligned}
$$

which is positive for small $\epsilon$. Therefore $E \cap B\left(b^{\prime}, \delta \rho\right) \neq \varnothing$, which proves the lemma.

For $A \subset R^{n}$, we set $A^{(1)}=\{2 a-b: a, b \in A\}$ and, when $k$ is an integer greater than one, $A^{(k)}=\left[A^{(k-1)}\right]^{(1)}$. 
The following two lemmas can be proved as Lemmas 2 and 3 in [3].

4.2. LEMmA. Suppose that $E$ is an $m$ regular subset of $R^{n}, \eta>0, \delta>0$ and $N$ is a positive integer. Then there are a positive number $d_{N}$ and an $m$ set $E_{N} \subset E$ such that $H^{m}\left(E \backslash E_{N}\right)<\eta$ and $A^{(N)} \subset E[\delta d(A)]$ whenever $A \subset$ $E_{N}$ and $d(A)<d_{N}$.

4.3. Lemma. Suppose that $A \subset R^{n}$ and

$$
x=\sum_{j=1}^{k} \beta_{j} a_{j}, \quad \sum_{j=1}^{k}\left|\beta_{j}\right| \leqslant 2 l+1
$$

where $a_{j} \in A, j=1, \cdots, k, \Sigma_{j=1}^{k} \beta_{j}=1$, the $\beta_{j}$ 's are integers precisely one of which is odd, and $l$ is a positive integer. Then $x \in A^{(l)}$.

If $A \subset R^{n}$, we denote by $\operatorname{Sp} A$ the smallest affine subspace of $R^{n}$ containing $A$.

4.4. Lemma. Suppose that $k$ is an integer and $\lambda$ and $p$ are real numbers such that $1 \leqslant k \leqslant n, 0<\lambda<1$ and $p>1$. Then there exists a positive integer $N=N(k, \lambda, p)$ with the property:

If $r>0, A=\left\{a_{0}, \cdots, a_{k}\right\} \subset R^{n},\left|a_{i}-a_{0}\right| \leqslant r$ and $d\left(a_{i}, \operatorname{Sp}\left\{a_{0}, \cdots, a_{i-1}\right\}\right)$ $\geqslant \lambda r$ for $i=1, \cdots, k$, then $(\operatorname{Sp} A) \cap B\left(a_{0}, p r\right) \subset A^{(N)}(k r)$.

Proof. Suppose that $r$ and $A=\left\{a_{0}, \cdots, a_{k}\right\}$ satisfy the above conditions. We may assume that $a_{0}=0$. Let $a \in(\operatorname{Sp} A) \cap B(0, p r)$. Then there are numbers $\alpha_{1}, \cdots, \alpha_{k}$ such that $a=\Sigma_{i=1}^{k} \alpha_{i} a_{i}$. Choose even integers $\beta_{1}, \cdots$, $\beta_{k}$ so that $\left|\alpha_{i}-\beta_{i}\right| \leqslant 1$ for $i=1, \cdots, k$ and put $b=\sum_{i=1}^{k} \beta_{i} a_{i}$. Then $|a-b| \leqslant k r$ and it suffices to show that $b \in A^{(N)}$ for some $N$ depending only on $k, \lambda$ and $p$. Write

$$
\beta_{0}=1-\sum_{i=1}^{k} \beta_{i}, \quad b=\sum_{i=0}^{k} \beta_{i} a_{i} .
$$

Let $e \in \operatorname{Sp} A$ be a unit vector perpendicular to $\operatorname{Sp}\left\{a_{0}, \cdots, a_{k-1}\right\}$. Then

$$
\left|a_{k} \cdot e\right|=d\left(a_{k}, \operatorname{Sp}\left\{a_{0}, \cdots, a_{k-1}\right\}\right) \geqslant \lambda r .
$$

Hence $p r \geqslant|a||e| \geqslant|a \cdot e|=\left|\alpha_{k} a_{k} \cdot e\right| \geqslant\left|\alpha_{k}\right| \lambda r$ and $\left|\alpha_{k}\right| \leqslant p / \lambda$. Replacing $a$ by $\sum_{i=1}^{k-1} \alpha_{i} a_{i}$ and observing that

$$
\left|\sum_{i=1}^{k-1} \alpha_{i} a_{i}\right| \leqslant|a|+\left|\alpha_{k}\right|\left|a_{k}\right| \leqslant \operatorname{pr}\left(1+\lambda^{-1}\right)
$$

we obtain in the same way as above that $\left|\alpha_{k-1}\right| \leqslant p\left(1+\lambda^{-1}\right) \lambda^{-1}$. Proceeding in this manner, we see that $\left|\alpha_{i}\right| \leqslant p\left(1+\lambda^{-1}\right)^{k-i} \lambda^{-1}$ for $i=1, \cdots, k$. 
Hence $\left|\alpha_{i}\right| \leqslant p 2^{k-1} \lambda^{-k}$ for $i=1, \cdots, k$. Therefore

$$
\sum_{i=1}^{k}\left|\beta_{i}\right| \leqslant \sum_{i=1}^{k}\left|\alpha_{i}\right|+\sum_{i=1}^{k}\left|\alpha_{i}-\beta_{i}\right| \leqslant k p 2^{k-1} \lambda^{-k}+k
$$

and

$$
\sum_{i=0}^{k}\left|\beta_{i}\right| \leqslant 1+2 \sum_{i=1}^{k}\left|\beta_{i}\right| \leqslant 1+2\left(k p 2^{k-1} \lambda^{-k}+k\right) \leqslant 1+2 N
$$

for $N \geqslant k p 2^{k-1} \lambda^{-k}+k$. The proof is complete by Lemma 4.3.

The following lemma can be proved as Lemma 5 in [3].

4.5. Lemma. There is a constant $K>1$ depending only on $n$ and $m$ such that for any $m$ regular set $E \subset R^{n}$ and for $\eta>0$ there is a positive number $d_{0}$ and an $m$-set $E_{0} \subset E$ with the properties:

(1) $H^{m}\left(E \backslash E_{0}\right)<\eta$.

(2) If $0<d<d_{0}, a \in E_{0}$ and $T \in A(a, n, m+1)$, then $T \cap B(a, K d)$ $\not \subset E_{0}(d)$.

4.6. Lemma. If $E$ is an $m$ regular subset of $R^{n}, \eta>0$ and $0<\lambda<1$, then there are a positive number $d^{*}$ and an $m$-set $E^{*} \subset E$ with the properties:

(1) $H^{m}\left(E \backslash E^{*}\right)<\eta$.

(2) If $a \in E^{*}$ and $0<r<d^{*}$, then there exists $V \in A(a, n, m)$ such that $E^{*} \cap[B(a, r) \backslash V(\lambda r)]=\varnothing$.

Proof. Let $K, d_{0}$ and $E_{0}$ be as in Lemma 4.5 and set $p=(n+2) K$. Next let $N=N(m+1, \lambda, p)$ be a positive integer having the property of Lemma 4.4. Taking $\delta=1$ in Lemma 4.2 and recalling 3.3, we find a positive number $d_{N}<d_{0}$ and an $m$-set $E_{N} \subset E_{0}$ such that $H^{m}\left(E \backslash E_{N}\right)<\eta$ and

$$
A^{(N)} \subset E_{0}[d(A)] \text { whenever } A \subset E_{N} \text { and } d(A)<d_{N} \text {. }
$$

We shall show that we may choose $d^{*}=(n+2)^{-1} d_{N}$ and $E^{*}=E_{N}$. Otherwise there are a point $a_{0} \in E_{N}$ and a positive number $r<(n+2)^{-1} d_{N}$ such that

$$
E_{N} \cap\left[B\left(a_{0}, r\right) \backslash V(\lambda)\right] \neq \varnothing \quad \text { for all } V \in A\left(a_{0}, n, m\right) .
$$

Take any $V_{0} \in A\left(a_{0}, n, m\right)$. Then there is $a_{1} \in E_{N} \cap\left[B\left(a_{0}, r\right) \backslash V_{0}(\lambda r)\right]$. Next take $V_{1} \in A\left(a_{0}, n, m\right) \cap A\left(a_{1}, n, m\right)$ and $a_{2} \in E_{N} \cap\left[B\left(a_{0}, r\right) \backslash V_{1}(\lambda)\right]$. Proceeding in this manner, we get the points $a_{0}, \cdots, a_{m+1}$ and the affine subspaces $V_{0}, \cdots, V_{m}$ such that

$$
a_{i} \in E_{N} \cap\left[B\left(a_{0}, r\right) \backslash V_{i-1}(\lambda r)\right] \text { for } i=1, \cdots, m+1 \text {, }
$$




$$
\left\{a_{0}, \cdots, a_{i}\right\} \subset V_{i} \text { for } i=0, \cdots, m \text {. }
$$

Hence $d\left(a_{i}, \operatorname{Sp}\left\{a_{0}, \cdots, a_{i-1}\right\}\right) \geqslant d\left(a_{i}, V_{i-1}\right)>\lambda$ for $i=1, \cdots, m+1$. Set $A=\left\{a_{0}, \cdots, a_{m+1}\right\}$ and $T=\operatorname{Sp} A$. Then $d(A) \leqslant 2 r<d_{N}$ and from 4.4 and (3) we obtain

$$
\begin{aligned}
T \cap B\left[a_{0}, K(n+2) r\right]=T & \cap B\left(a_{0}, p r\right) \\
& \subset A^{(N)}[(m+1) r] \subset E_{0}[(m+3) r] \subset E_{0}[(n+2) r] .
\end{aligned}
$$

Taking $d=(n+2) r<d_{N}<d_{0}$, we arrive at a contradiction by Lemma 4.5.

4.7. Lemma. Let $E$ be an $m$-set in $R^{n}$ and $\epsilon>0$. Then there are a positive number $d$ and an m-set $E_{0} \subset E$ such that if $a \in R^{n}, V \in A(a, n, m)$, $B \subset V$ and $0<h<l<d$, then

$$
H^{m}\left[E_{0} \cap P_{V}^{-1}(B) \cap V(h)\right] \leqslant(1+\epsilon)\left(1+h / b^{m} H^{m}[B(l) \cap \eta] .\right.
$$

In the proof we may assume that $V=\left\{x: x_{m+1}=\cdots=x_{n}=0\right\}$. We can then carry out the proof as the proof of Lemma 7 in [3] when we replace the cylinders $C(x, y)$ by the sets $C\left(x_{1}, \cdots, x_{m}\right)=V(h) \cap\left\{y:\left(x_{1}-y_{1}\right)^{2}+\right.$ $\left.\cdots+\left(x_{m}-y_{m}\right)^{2}<l^{2}\right\}$ and note that $d\left[C\left(x_{1}, \cdots, x_{m}\right)\right]<2(l+h)$.

4.8. LEMMA. Let $E$ be an $m$ regular subset of $R^{n}$ and $0<\mu<1$. Then there are an $m$-set $E_{1} \subset E$ and a positive number $d_{1}$ with the property: If $a \in E_{1}$ and $0<r<d_{1}$, then there exists $V \in A(a, n, m)$ such that

$$
\begin{gathered}
E_{1} \cap[B(a, r) \backslash V(\mu r)]=\varnothing, \\
V \cap B(a, r) \subset E(\mu r) .
\end{gathered}
$$

Proof. Let $0<\epsilon<1 / 2$. Using 4.7, 4.6 and 3.3, we can find an $m$-set $E_{1} \subset E$ and a positive number $d_{1}$ with the properties:

If $a \in R^{n}, V \in A(a, n, m), B \subset V$ and $0<h<l<d_{1}$, then

$$
H^{m}\left[E_{1} \cap P_{V}^{-1}(B) \cap V(h)\right] \leqslant(1+\epsilon)(1+h / l)^{m} H^{m}[B(l) \cap V] \text {. }
$$

If $a \in E_{1}$ and $0<r<d_{1}$, then there is $V \in A(a, n, m)$ such that

(5) $\quad H^{m}\left[E_{1} \cap B(a, r)\right]>(1-\epsilon) \alpha(m) r^{m}$ if $a \in E_{1}$ and $0<r<d_{1}$.

Let $a \in E_{1}, 0<r<d_{1}$ and take $V$ as in (4). Then (1) follows from (4). Suppose that (2) is false. Then there is a point $b \in V \cap B(a, r)$ such that $B(b, \mu r) \cap E=\varnothing$. Set $B=V \cap[B(a, r) \backslash B(b, \mu r / 2)]$. Then, since $\epsilon^{2}<1 / 2$,

$$
E_{1} \cap V\left(\epsilon^{2} \mu r\right) \cap B(a, r) \subset E_{1} \cap V\left(\epsilon^{2} \mu r\right) \cap P_{V}^{-1}(B) .
$$


Hence by (4) and (5),

$$
H^{m}\left[E_{1} \cap V\left(\epsilon^{2} \mu r\right) \cap P_{V}^{-1}(B)\right] \geqslant H^{m}\left[E_{1} \cap B(a, r)\right]>(1-\epsilon) \alpha(m) r^{m} .
$$

From the definition of $B$, it follows that

$$
H^{m}[B(\epsilon \mu r) \cap V] \leqslant \alpha(m)\left[(1+\epsilon \mu)^{m}-2^{-m}(1 / 2-\epsilon)^{m} \mu^{m}\right] r^{m} .
$$

These inequalities together with (3) give

$$
(1-\epsilon) \alpha(m) r^{m}<(1+\epsilon)^{m+1} \alpha(m)\left[(1+\epsilon \mu)^{m}-2^{-m}(1 / 2-\epsilon)^{m} \mu^{m}\right] r^{m} .
$$

For small $\epsilon$ this is false, and the proof is complete.

5. Projection properties of $m$ regular sets. In this section we shall prove two lemmas from which Theorem 2.1 easily follows. Actually these lemmas say more than we need. Instead of proving Lemma 5.1, we could use a result of Federer (see [2, 3.3.12 and 2.10.11]) which implies that if $E$ is a purely $m$ unrectifiable $m$-set, then $H^{m}\left[P_{V}(E)\right]=0$ for almost all $V \in G(n, m)$. This is a rather deep result and we want to give a proof independent of it. Instead of Lemma 5.2 it would be sufficient to show that if $E$ is $m$ regular, then $H^{m}\left[P_{V}(E)\right]>0$ for some $V \in G(n, m)$, but the proof would be almost the same. After one knows that $E$ is $m$ rectifiable, one can prove statements that are stronger than Lemma 5.2 using known properties of $m$ rectifiable sets (e.g. $[2,3.2 .29])$.

5.1. Lemma. If $E$ is a purely $m$ unrectifiable $m$ regular subset of $R^{n}$ and $V \in G(n, m)$, then $H^{m}\left[P_{V}(E)\right]=0$.

Proof. Let $0<\epsilon<1 / 2$. Using the regularity of $E$ and Lemma 4.6, we can find a positive number $d^{*}$ and an $m$-set $E^{*} \subset E$ with the properties:

$$
H^{m}\left(E \backslash E^{*}\right)<\epsilon .
$$

If $a \in E^{*}$ and $0<r<d^{*}$, then

$$
H^{m}[E \cap B(a, r)]>\alpha(m) r^{m} / 2,
$$

and there is $W \in A(a, n, m)$ such that

$$
E^{*} \cap[B(a, r) \backslash W(\epsilon r)]=\varnothing .
$$

Since $E$ is purely $m$ unrectifiable, it follows from $[2,3.3 .5]$ that for $H^{m}$ almost every $a \in E^{*}$, there are points $b \in E^{*}$ arbitrarily close to $a$ such that $\left|P_{V}(b-a)\right|<\epsilon|b-a|$.

Suppose now that $a, b \in E^{*}$ satisfy the above inequality and that $r=$ $|a-b|<d^{*}$. Choose $W$ as in (3) and let $c=P_{W}(b)$. Using (3) and the basic properties of orthogonal projection, we see that 


$$
|c-b| \leqslant \epsilon r, \quad r / 2 \leqslant|c-a| \leqslant r, \quad\left|P_{V}(c-a)\right|<2 \epsilon r .
$$

By $[2,1.7 .3]$, we can choose an orthonormal base $\left\{e_{1}, \cdots, e_{m}\right\}$ for $\{x$ : $x+a \in W$ \} such that $P_{V}\left(e_{i}\right) \cdot P_{V}\left(e_{j}\right)=0$ for $i \neq j$. Then, for some $i=i_{0}$,

$$
\left|P_{V}\left(e_{i_{0}}\right)\right|<2 r^{-1}\left|P_{V}(c-a)\right|<4 \epsilon,
$$

because otherwise we should have

$$
\begin{aligned}
\left|P_{V}(c-a)\right|^{2} & =\sum_{i=1}^{m}\left|(c-a) \cdot e_{i}\right|^{2}\left|P_{V}\left(e_{i}\right)\right|^{2} \\
& >4 r^{-2}\left|P_{V}(c-a)\right|^{2}|c-a|^{2} \geqslant\left|P_{V}(c-a)\right|^{2}
\end{aligned}
$$

It follows that $\boldsymbol{P}_{V}$ maps $W \cap B(a, r)$ into an $m$-dimensional rectangle whose one side has length $8 \epsilon r$ and the others $2 r$. Hence (3) implies that $P_{V}\left[E^{*} \cap B(a, r)\right]$ is contained in a rectangle with side lengths $10 e r, 2 r+2 \epsilon r, \cdots, 2 r+2 \epsilon r$. Therefore

$$
H^{m}\left(P_{V}\left[E^{*} \cap B(a, r)\right]\right)<10^{m} \epsilon r^{m} .
$$

The inequality (4) holds at $H^{m}$ almost all points $a \in E^{*}$ with arbitrarily small values of $r$. By the covering theorem $[2,2.8 .15]$, we can cover almost all of $E^{*}$ with disjoint balls $B\left(a_{j}, r_{j}\right), j=1,2, \cdots$, for which (4) is valid and $a_{j} \in E^{*}$. Using (4) and (2), we obtain

$$
\begin{aligned}
H^{m}\left[P_{V}\left(E^{*}\right)\right] & \leqslant \sum_{j=1}^{\infty} H^{m}\left(P_{V}\left[E^{*} \cap B\left(a_{j}, r_{j}\right)\right]\right) \leqslant 10^{m} \in \sum_{j=1}^{\infty} r_{j}^{m} \\
& <10^{m} \cdot 2 \alpha(m)^{-1} \epsilon \sum_{j=1}^{\infty} H^{m}\left[E \cap B\left(a_{j}, r_{j}\right)\right] \\
& \leqslant 10^{m} \cdot 2 \alpha(m)^{-1} \epsilon H^{m}(E) .
\end{aligned}
$$

From (1) we get

$$
H^{m}\left[P_{V}\left(E \backslash E^{*}\right)\right] \leqslant H^{m}\left(E \backslash E^{*}\right)<\epsilon,
$$

and finally

$$
H^{m}\left[P_{V}(E)\right]<\epsilon\left[1+10^{m} \cdot 2 \alpha(m)^{-1} H^{m}(E)\right],
$$

which proves the lemma.

5.2. LEMMA. If $E$ is an $m$ regular subset of $R^{n}$, then

$$
\lim _{r \rightarrow 0+} \sup _{V \in A(a, n, m)} \frac{H^{m}\left(P_{V}[E \cap B(a, r)]\right)}{\alpha(m) r^{m}}=1
$$

for $H^{m}$ a.e. $a \in E$. 
Proof. The left-hand side of the asserted equation with "lim" replaced by "lim sup" is never greater than the upper density of $E$, whence it is at most one $H^{m}$ almost everywhere in $E$. Suppose that the lemma is false. Then we can find an $m$ regular set $E$ and a number $\eta, 0<\eta<1$, such that

$$
\liminf _{r \rightarrow 0+} \sup _{V \in A(a, n, m)} \frac{H^{m}\left(P_{V}[E \cap B(a, r)]\right)}{\alpha(m) r^{m}}<\eta
$$

for all $a \in E$. This follows from the fact that the function whose value at $a$ is the left-hand side of (1) is Borel measurable, as one can see by standard methods (cf. $[1,3.4]$ ). By regularity, there are a positive number $d_{0}$ and an $m$-set $E_{0} \subset$ $E$ such that

$$
H^{m}[E \cap B(a, r)]>\alpha(m) r^{m} / 2 \text { if } a \in E_{0} \text { and } 0<r<d_{0} .
$$

Denote $t=[(\eta+1) / 2]^{1 / m}$ and let $0<\mu<(1-t) / 16$ and $\epsilon>0$. Since every $m$-set contains a closed $m$-set by [2, 2.2.2(1)], we can apply Lemma 4.8 to get a positive number $d_{1}<d_{0}$ and a closed $m$-set $E_{1} \subset E_{0}$ such that if $a \in E_{1}$ and $0<r<d_{1}$, then there is $V \in A(a, n, m)$ for which

$$
E_{1} \cap[B(a, r) \backslash V(\mu r)]=\varnothing,
$$

$$
V \cap B(a, r) \subset E_{0}(\mu r) \text {. }
$$

For $H^{m}$ a.e. $x \in E_{1}$, we have $\Theta^{m}(E, x)=1$ and $\Theta^{m}\left(E \backslash E_{1}, x\right)=0$ because of the regularity of $E$ and 3.2. Therefore, using again Lemma 4.8, we can find a point $a_{0} \in E_{1}$, a positive number $r_{0}<d_{1}$ and an affine subspace $V_{0} \in$ $A\left(a_{0}, n, m\right)$ such that

$$
\begin{gathered}
H^{m}\left[E \cap B\left(a_{0}, r_{0}\right)\right]<2 \alpha(m) r_{0}^{m}, \\
H^{m}\left[\left(E \backslash E_{1}\right) \cap B\left(a_{0}, r_{0}\right)\right]<\epsilon \alpha(m) r_{0}^{m}, \\
\alpha(m)^{-1} r_{0}^{-m} H^{m}\left(P_{V_{0}}\left[E \cap B\left(a_{0}, r_{0}\right)\right]\right)<\eta, \\
E_{1} \cap\left[B\left(a_{0}, r_{0}\right) \backslash V_{0}\left(\mu r_{0}\right)\right]=\varnothing, \\
V_{0} \cap B\left(a_{0}, r_{0}\right) \subset E_{1}\left(\mu r_{0}\right) .
\end{gathered}
$$

We denote $P=P_{V_{0}}$ and set

$$
F=P\left[E_{1} \cap B\left(a_{0}, r_{0}\right)\right] \text { and } G=\left[V_{0} \cap B\left(a_{0}, t r_{0}\right)\right] \backslash F .
$$

Then $F$ is closed and $H^{m}(F) \leqslant \eta \alpha(m) r_{0}^{m}$ by (7), whence 


$$
H^{m}(G) \geqslant\left(t^{m}-\eta\right) \alpha(m) r_{0}^{m}=(1-\eta) \alpha(m) r_{0}^{m} / 2 .
$$

We can cover $G$ with balls $B(b, \rho)$ such that $b \in G, F \cap U(b, \rho)=\varnothing$ and $F \cap \partial B(b, \rho) \neq \varnothing$. Then, using e.g. the covering lemma in $[6$, p. 9] or $[2,2.8 .5]$, we can select a finite number of them, say $B\left(b_{q}, \rho_{q}\right), q=1, \cdots, l$, such that

$$
B\left(b_{q}, 5 \rho_{q}\right) \cap B\left(b_{q^{\prime}}, 5 \rho_{q^{\prime}}\right)=\varnothing \quad \text { for } q \neq q^{\prime}
$$

and

$$
\sum_{q=1}^{l} \rho_{q}^{m}>K_{1} r_{0}^{m}
$$

where $K_{1}$ is a positive constant depending only on $m$ and $\eta$. From (9) and the inequality $\mu r_{0}<(1-t) r_{0}$, we obtain

$$
\rho_{q} \leqslant \mu r_{0} \text { for } q=1, \cdots, l \text {. }
$$

We now consider the sets $C_{q}=P^{-1}\left[B\left(b_{q}, \rho_{q} / 2\right)\right] \cap V_{0}\left[(1-t) r_{0} / 2\right]$ and rearrange them so that they contain no points of $E_{0}$ for $q=1, \cdots, k$ and that they contain at least one point, $c_{q}$, of $E_{0}$ for $q=k+1, \cdots, l$. If $x \in$ $E \cap B\left(c_{q}, \rho_{q} / 4\right)$ for some $q=k+1, \cdots, l$, then using the facts that $b_{q} \in$ $B\left(a_{0}, t r_{0}\right), c_{q} \in C_{q},(12)$ and $\mu<(1-t) / 3$, we can infer that $x \in B\left(a_{0}, r_{0}\right)$ and $P(x) \in U\left(b_{q}, \rho_{q}\right)$, whence $P(x) \notin F$ and $x \notin E_{1}$. Therefore

$$
\bigcup_{q=k+1}^{l} E \cap B\left(c_{q}, \rho_{q} / 4\right) \subset\left(E \backslash E_{1}\right) \cap B\left(a_{0}, r_{0}\right) .
$$

Since the balls $B\left(c_{q}, \rho_{q} / 4\right)$ are disjoint by (10), we obtain from (2), (6) and (13) that

$$
2^{-2 m-1} \alpha(m) \sum_{q=k+1}^{l} \rho_{q}^{m}<\epsilon \alpha(m) r_{0}^{m}
$$

and taking $\epsilon<2^{-2 m-2} K_{1}$, as we may, we get from this and (11)

$$
\sum_{q=1}^{k} \rho_{q}^{m}>K_{1} r_{0}^{m} / 2
$$

From now on we only consider $q=1, \cdots, k$. Recalling (8) and how the balls $B\left(b_{q}, \rho_{q}\right)$ were chosen, we see that there are points $e_{q}$ such that

$$
e_{q} \in P^{-1}\left[\partial B\left(b_{q}, \rho_{q}\right)\right] \cap V_{0}\left(\mu r_{0}\right) \cap E_{1} \text { for } q=1, \cdots, k \text {. }
$$

By (12), $\mu^{-1} \rho_{q} \leqslant r_{0}<d_{1}$, and we can apply (4) to get $V_{q} \in A\left(e_{q}, n, m\right)$ such that

$$
A_{q} \subset E_{0}\left[(1-t) \rho_{q} / 8\right] \text { for } q=1, \cdots, k,
$$

where we have denoted $A_{q}=B\left[e_{q}, \mu^{-1}(1-t) \rho_{q} / 8\right] \cap V_{q}$. 
We show that $b_{q} \notin P\left(A_{q}\right)$. If there is a point $f_{q} \in A_{q}$ such that $P\left(f_{q}\right)=$ $b_{q}$, then it follows from (15) and (12) that $f_{q} \in B\left[b_{q},(1-t) r_{0} / 4\right]$ and by (16) there is $x_{q} \in E_{0} \cap B\left[f_{q},(1-t) \rho_{q} / 8\right]$. Then $x_{q} \in E_{0} \cap B\left[b_{q},(1-t) r_{0} / 2\right]$ and $\left|P\left(x_{q}\right)-b_{q}\right|<(1-t) \rho_{q} / 8$. Hence $x_{q} \in E_{0} \cap C_{q}=\varnothing$, which is a contradiction.

Let $I_{q}$ be the closed segment with end points $b_{q}$ and $P\left(e_{q}\right)$. Then $I_{q} \cap \partial_{V_{0}} P\left(A_{q}\right) \neq \varnothing$, since $I_{q}$ is connected, $b_{q} \in I_{q} P\left(A_{q}\right)$ and $P\left(e_{q}\right) \in I_{q} \cap$ $P\left(A_{q}\right)$. Here $\partial_{V_{0}}$ means the boundary relative to $V_{0}$. But since $P \mid V_{q}$ is composed of two translations and a linear map between two $m$-dimensional linear subspaces of $R^{n}$, it follows that $\partial_{V_{0}} P\left(A_{q}\right)=P\left(\partial_{V_{q}} A_{q}\right)$. Hence there is a point $a_{q} \in \partial_{V_{q}} A_{q}$ such that $P\left(a_{q}\right) \in I_{q}$. Let $J_{q}$ be the closed segment between $e_{q}$ and $a_{q}$. Then $J_{q} \subset A_{q}$ and $P\left(J_{q}\right) \subset I_{q}$ and thus by (15)

$$
\left|P(x)-b_{q}\right| \leqslant \rho_{q} \text { whenever } x \in J_{q} \text {. }
$$

By (16), $J_{q}$ can be covered with balls $B\left(x, \rho_{q}\right)$ where $x \in E_{0}$. We can choose a finite number of them, say $B\left(x_{q j}, \rho_{q}\right), j=1, \cdots, s$, so that

$$
\begin{aligned}
J_{q} \cap B\left(x_{q j}, \rho_{q}\right) & \neq \varnothing \quad \text { for } j=1, \cdots, s, \\
B\left(x_{q i}, 2 \rho_{q}\right) \cap B\left(x_{q j}, 2 \rho_{q}\right) & =\varnothing \quad \text { for } i \neq j, i, j=1, \cdots, s, \\
s & >K_{2} \mu^{-1},
\end{aligned}
$$

where $K_{2}$ is a constant depending only on $t$. This can be seen by choosing a maximal set with respect to the properties (18) and (19) and using the fact that the length of $J_{q}$ is $\mu^{-1}(1-t) \rho_{q} / 8$.

Set

$$
B_{q}=\bigcup_{j=1}^{s} B\left(x_{q j}, 2 \rho_{q}\right) \text { for } q=1, \cdots, k .
$$

We shall show that the sets $B_{q}$ are disjoint and that they are contained in $B\left(\dot{a}_{0}, r_{0}\right)$. To do this, consider any $q=1, \cdots, k$ and let $x \in B_{q}$. Suppose that $x \in B\left(x_{q j}, 2 \rho_{q}\right)$. Then by (18) there is $y \in J_{q}$ such that $\left|x_{q j}-y\right| \leqslant \rho_{q}$. Using triangle inequality and (17), we see that $\left|P(x)-b_{q}\right| \leqslant 4 \rho_{q}$, and this together with (10) implies that the sets $B_{q}, q=1, \cdots, k$, are disjoint. Applying triangle inequality to the points $x, x_{q j}, y, e_{q}, P\left(e_{q}\right), b_{q}$ and $a_{0}$ and using (15) and (12), we find that $\left|x-a_{0}\right|<r_{0}$. Hence $\bigcup_{q=1}^{k} B_{q} \subset B\left(a_{0}, r_{0}\right)$.

We deduce from (19), (2) and (20) that

$$
\begin{aligned}
H^{m}\left(E \cap B_{q}\right) & =\sum_{j=1}^{s} H^{m}\left[E \cap B\left(x_{q j}, 2 \rho_{q}\right)\right] \\
& >s 2^{-1} \alpha(m)\left(2 \rho_{q}\right)^{m}>2^{m-1} \alpha(m) K_{2} \mu^{-1} \rho_{q}^{m}
\end{aligned}
$$


for all $q=1, \cdots, k$. Therefore (14) gives

$$
\begin{aligned}
H^{m}\left[E \cap B\left(a_{0}, r_{0}\right)\right] & \geqslant \sum_{q=1}^{k} H^{m}\left(E \cap B_{q}\right) \\
& >2^{m-1} \alpha(m) K_{2} \mu^{-1} \sum_{q=1}^{k} \rho_{q}^{m}>2^{m-2} \alpha(m) K_{1} K_{2} \mu^{-1} r_{0}^{m} .
\end{aligned}
$$

But this contradicts with (5) if we choose, as we may, $\mu<2^{m-3} K_{1} K_{2}$. This completes the proof.

Proof of Theorem 2.1. We shall show that we may assume $E$ to be $m$ regular. First, we can cover $H^{m}$ almost all of $E$ with countably many open balls $U_{i}$ such that $H^{m}\left(E \cap U_{i}\right)<\infty$. Then $\Theta^{m}\left(E \cap U_{i}, x\right)=\Theta^{m}(E, x)=1$ for $H^{m}$ a.e. $x \in E \cap U_{i}$. Hence we may assume that $H^{m}(E)<\infty$.

Secondly, suppose that $H^{m}(E)<\infty$. The set $E^{\prime}=\left\{x \in R^{n}: \Theta^{m}(E, x)=\right.$ 1\} is a Borel set (see $[1,3.4]), H^{m}\left(E \backslash E^{\prime}\right)=0$ and $H^{m}\left(E^{\prime}\right)=H^{m}(E)<\infty$ by $[2,2.10 .19(3)]$. Therefore 3.2 implies that $\Theta^{* m}\left(E^{\prime}, x\right) \leqslant 1$ for $H^{m}$ a.e. $x \in E^{\prime}$. We also have $\Theta_{*}^{m}\left(E^{\prime}, x\right) \geqslant \Theta_{*}^{m}(E, x)=1$ for all $x \in E^{\prime}$. Hence $\Theta^{m}\left(E^{\prime}, x\right)=1$ for $H^{m}$ a.e. $x \in E^{\prime}$, and we may assume that $E$ is $m$ regular.

If $E$ were $m$ regular and not $m$ rectifiable, it would contain a purely $m$ unrectifiable $m$-set $B$, see $[2,3.2 .14]$. By $3.3, B$ would be both $m$ regular and purely $m$ unrectifiable, but because of 5.1 and 5.2 such a set cannot exist. The theorem is proved.

\section{REFERENCES}

1. H. Federer, The $(\phi, k)$ rectifiable subsets of $n$ space, Trans. Amer. Math. Soc. 62 (1947), 114-192. MR 9, 231.

2. - Geometric measure theory, Die Grundlehren der math. Wissenschaften, Band 153, Springer-Verlag, New York, 1969. MR 41 \#1976.

3. J. M. Marstrand, Hausdorff two-dimensional measure in 3-space, Proc. London Math. Soc. (3) 11 (1961), 91-108. MR 23 \#A994.

4. E. F. Moore, Density ratios and $(\phi, 1)$ rectifiability in n-space, Trans. Amer. Math. Soc. 69 (1950), 324-334. MR 12, 324.

5. A. P. Morse and J. F. Randolph, The $\phi$ rectifiable subsets of the plane, Trans. Amer. Math. Soc. 55 (1944), 236-305. MR 5, 232.

6. E. M. Stein, Singular integrals and differentiability properties of functions, Princeton, Math. Series, no. 30, Princeton Univ. Press, Princeton, N. J., 1970. MR 44 \#7280. INDIANA 47401

Current address: Department of Mathematics, University of Helsinki, Helsinki, Finland 